\title{
Comunicação
}

[Communication]

\section{Precisão da técnica de absorciometria de raios-x de dupla energia na determinação da composição corporal em gatos}

[Precision of dual energy $x$-ray absorptiometry for body composition measurements in cats]

\author{
N.C. Borges ${ }^{1}$, R.S. Vasconcellos ${ }^{2}$, J.C. Canola ${ }^{2}$, F.J.A. Paula ${ }^{3}$, A.C. Carciofi $^{2}$, G.T. Pereira ${ }^{2}$ \\ ${ }^{1}$ Escola de Veterinária - UFG \\ Caixa Postal 131 \\ 74001-970 - Goiânia, GO \\ ${ }^{2}$ Faculdade de Ciências Agrárias e Veterinárias - UNESP - Jaboticabal, SP \\ ${ }^{3}$ Faculdade de Medicina - USP - Ribeirão Preto, SP
}

A técnica de absorciometria de raios- $x$ de dupla energia (DEXA) representa significativo aperfeiçoamento no campo de diagnóstico por imagem, uma vez que a fonte de radiação é mais estável, e a diferença entre os níveis de radiação $(40 \mathrm{kV}$ e $70 \mathrm{kV})$ emitidos permite melhor resolução (Pietrobelli et al., 1996).

Embora consagrada como técnica precisa e exata, é necessário conhecer o grau de erro para cada espécie, em relação ao aparelho, ao posicionamento do indivíduo e as variações intra-operadores. Glüer et al. (1995) afirmaram que $\mathrm{o}$ erro refere-se à característica de reprodutibilidade de uma técnica e pode ser avaliado em curto ou longo prazo. O erro de precisão em curto prazo referente à técnica é calculado considerando o coeficiente de variação das médias de varreduras individuais e observando o pool de varreduras em vários indivíduos.

Neste estudo a precisão da técnica de $\operatorname{DEXA}^{1}$ foi determinada em quatro gatos mestiços adultos gonadectomizados, com peso corporal (PC) médio $4342 \mathrm{~g}$ e três fêmeas com $\mathrm{PC}$ de $3459 \mathrm{~g}^{2}$. Os animais passaram por avaliações periódicas e

Recebido em 24 de maio de 2007

Aceito em 11 de dezembro de 2007

E-mail: naida@vet.ufg.br

${ }^{1}$ QDR 4500 Elite Windows ${ }^{\circledR}$, Version Student's Guide

Hologic, Inc. 35. - EUA.

${ }^{2}$ Aprovado pelo Comitê de Ética da FCAV-UNESP -

Jaboticabal, SP - Brasil. foram alimentados com ração seca comercial $^{3} \mathrm{e}$ água.

O exame de DEXA foi realizado no setor de imagens do Hospital das Clínicas - FM-USP Ribeiro Preto, e as imagens analisadas para o corpo total com o uso do software pediátrico 4 .

Antes do exame, os animais foram anestesiados com a associação de cloridratos de levomepromazina $^{5}$, de tiletamina e de zolazepam $^{6}$ nas doses respectivas de 0,$5 ; 2,5$ e $2,5 \mathrm{mg} / \mathrm{kg}$ por $\mathrm{PC}$, por via intramuscular, numa mesma seringa. Após perda do reflexo postural, os animais foram posicionados em decúbito dorsal com os membros torácicos e pélvicos estendidos no sentido caudal, e o correto posicionamento, sobre a mesa, foi mantido aplicando-se fitas adesivas ${ }^{7}$ (Lauten et al., 2000). Inicialmente foram realizadas cinco varreduras consecutivas sem reposicionamento (SR) do animal. Na seqüência, eram retiradas todas as fitas adesivas, e o animal era novamente posicionado para outras cinco varreduras com reposicionamento (CR) entre as mesmas.

\footnotetext{
${ }^{3}$ Guabi natural gatos adultos - Mogiana Alimentos S/A Campinas, Brasil.

${ }^{4}$ Pediatric whole body QDR Series uses QDR for Windows TM running on the Windows ${ }^{\circledR} 98$ System.

${ }^{5}$ Neozine - Aventis Pharma LTDA - São Paulo, Brasil.

${ }^{6}$ Zoletil - Virbac do Brasil Indústria e Comércio LTDA - São Paulo, Brasil.

${ }^{7}$ Esparadrapo Impermeável - Cremer S.A. - Blumenau, Brasil.
} 
Para determinar o erro de precisão individual, foram calculados o desvio-padrão (S) e o coeficiente de variação (CV) das médias das varreduras segundo Bussab e Moretin (2003). As médias das varreduras $\mathrm{CR}$ e $\mathrm{SR}$ foram comparadas por meio do teste $\mathrm{t}$ de Student para amostras pareadas com $5 \%$ de significância, utilizando software estatístico $\mathrm{SAS} \circledast$ (Schlotzhaver e Littel, 1997). O percentual do erro de precisão foi expresso pelo $\mathrm{CV}$ da técnica $\left(\mathrm{CV}_{\mathrm{t}}\right)$, e o grau de liberdade (GL) foi calculado de acordo com Glüer et al. (1995). Os valores de variância $\left(\mathrm{S}^{2}\right)$ foram comparados pelo teste $\mathrm{F}$ de Fisher, e o intervalo de confiança (IC) calculado de acordo com Bussab e Moretin (2003).

$\mathrm{Na}$ Tab. 1, estão representados os resultados do erro de precisão individual. Os maiores $\mathrm{CV}$ ocorreram no grupo $\mathrm{CR}$ e essa variabilidade pode ser explicada pelo somatório de efeitos, animal e equipamento de acordo com a citação de Glüer et al. (1995). O PC médio dos animais não diferiu entre os tratamentos, porém, no grupo $\mathrm{CR}$, as médias de conteúdo mineral ósseo (CMO), massa magra (MM) e massa gorda (MG) foram significativamente $(\mathrm{P}>0,05)$ maiores. Esses resultados indicam que o reposicionamento do animal entre as varreduras interfere nos valores de algumas variáveis e ressaltam a variabilidade de conformação corporal dos indivíduos. Os CV mínimos e máximos para as variáveis $\mathrm{CMO}$, densidade mineral óssea (DMO), MM e MG foram semelhantes aos obtidos por Munday et al. (1994) e Lauten et al. (2000). Ressalta-se, entretanto, que Lauten et al. (2000) trabalharam com um único gato e, portanto, não analisaram a variação corporal entre animais. De acordo com Glüer et al. (1995), mensurações feitas em um único indivíduo não representam o desempenho geral da técnica e, por conseguinte, não expressam adequadamente o erro de precisão em curto prazo.

Tabela 1. Médias, desvios-padrão (S) e coeficientes de variação (CV) obtidos com (CR) e sem (SR) reposicionamento de gatos adultos $(\mathrm{n}=7)$ sobre a mesa de DEXA

\begin{tabular}{|c|c|c|c|c|c|c|c|c|c|c|c|}
\hline \multirow{2}{*}{ Animal } & \multirow{2}{*}{ Estatística } & \multicolumn{2}{|c|}{ Peso (g) } & \multicolumn{2}{|c|}{ CMO (g) } & \multicolumn{2}{|c|}{$\mathrm{DMO}\left(\mathrm{g} / \mathrm{cm}^{2}\right)$} & \multicolumn{2}{|c|}{ MM (g) } & \multicolumn{2}{|c|}{ MG (g) } \\
\hline & & CR & $\overline{S R}$ & $\overline{\mathrm{CR}}$ & $\overline{S R}$ & $\overline{C R}$ & $\overline{S R}$ & $\overline{\mathrm{CR}}$ & SR & $\overline{\mathrm{CR}}$ & SR \\
\hline \multirow[t]{2}{*}{1} & $\mathrm{M} \pm \mathrm{S}$ & $4094,1 \pm 14,3$ & $4113,5 \pm 17,9$ & $134,8 \pm 3,6$ & $126,3 \pm 2,2$ & $0,470 \pm 0,006$ & $0,461 \pm 0,003$ & $2513,5 \pm 64,8$ & $2410,9 \pm 65,8$ & $1445,7 \pm 74,7$ & $1476,3 \pm 83,4$ \\
\hline & $\mathrm{CV}(\%)$ & 0,3 & 0,4 & 2,6 & 1,8 & 1,3 & 0,7 & 2,6 & 2,7 & 5,2 & 5,3 \\
\hline \multirow[t]{2}{*}{2} & $\mathrm{M} \pm \mathrm{S}$ & $2863,0 \pm 7,2$ & $2858,5 \pm 8,2$ & $71,3 \pm 3,2$ & $65,3 \pm 1,5$ & $0,382 \pm 0,016$ & $0,368 \pm 0,004$ & $1985,0 \pm 24,6$ & $1921,4 \pm 6,1$ & $806,8 \pm 26,1$ & $801,7 \pm 5,9$ \\
\hline & CV(\%) & 0,3 & 0,3 & 5,7 & 2,3 & 4,3 & 1,2 & 1,2 & 0,3 & 3,2 & 1,7 \\
\hline \multirow[t]{2}{*}{3} & $\mathrm{M} \pm \mathrm{S}$ & $3830,6 \pm 28,5$ & $3749,4 \pm 7,1$ & $99,5 \pm 3,0$ & $94,3 \pm 1,3$ & $0,425 \pm 0,005$ & $0,425 \pm 0,003$ & $2790,0 \pm 127,7$ & $2694,4 \pm 90,2$ & $941,0 \pm 149,4$ & $910,8 \pm 96,2$ \\
\hline & CV(\%) & 0,7 & 0,2 & 3,0 & 1,4 & 1,2 & 0,8 & 4,6 & 3,3 & 15,9 & 10,0 \\
\hline \multirow[t]{2}{*}{4} & $\mathrm{M} \pm \mathrm{S}$ & $4224,5 \pm 13,8$ & $4262,2 \pm 20,0$ & $145,5 \pm 5,0$ & $144,2 \pm 1,5$ & $0,485 \pm 0,008$ & $0,485 \pm 0,007$ & $2806,9 \pm 104,9$ & $2720,2 \pm 115,1$ & $1272,0 \pm 117,4$ & $1217,8 \pm 133,7$ \\
\hline & $\mathrm{CV}(\%)$ & 0,3 & 0,5 & 3,4 & 1,0 & 1,5 & 1,4 & 3,7 & 3,2 & 9,2 & 9,6 \\
\hline \multirow[t]{2}{*}{5} & $\mathrm{M} \pm \mathrm{S}$ & $5007,6 \pm 40,5$ & $4995,8 \pm 49,4$ & $164,5 \pm 3,1$ & $165,0 \pm 3,3$ & $0,496 \pm 0,009$ & $0,503 \pm 0,006$ & $3406,5 \pm 135,1$ & $3337,9 \pm 122,3$ & $1436,6 \pm 172,7$ & $1412,9 \pm 165,7$ \\
\hline & $\mathrm{CV}(\%)$ & 0,8 & 1,0 & 1,9 & 2,0 & 1,8 & 1,2 & 4,0 & 3,7 & 12,0 & 11,1 \\
\hline \multirow[t]{2}{*}{6} & $\mathrm{M} \pm \mathrm{S}$ & $4027,7 \pm 59,2$ & $4010,9 \pm 45,6$ & $95,7 \pm 3,6$ & $97,3 \pm 5,5$ & $0,421 \pm 0,019$ & $0,412 \pm 0,014$ & $2668,7 \pm 57,5$ & $2622,9 \pm 49,3$ & $1263,3 \pm 79,9$ & $1225,7 \pm 61,4$ \\
\hline & $\mathrm{CV}(\%)$ & 1,5 & 1,1 & 3,7 & 2,6 & 4,4 & 1,5 & 2,2 & 1,9 & 6,3 & 4,8 \\
\hline \multirow[t]{2}{*}{7} & $\mathrm{M} \pm \mathrm{S}$ & $3714,8 \pm 20,8$ & $3736,7 \pm 15,2$ & $81,8 \pm 3,3$ & $77,7 \pm 1,1$ & $0,389 \pm 0,029$ & $0,401 \pm 0,004$ & $2336,4 \pm 186,8$ & $2258,1 \pm 47,5$ & $1296,6 \pm 208,5$ & $1206,5 \pm 50,5$ \\
\hline & $\mathrm{CV}(\%)$ & 0,6 & 0,4 & 4,0 & 1,4 & 7,5 & 1,1 & 8,0 & 2,1 & 16,1 & 3,6 \\
\hline \multicolumn{2}{|c|}{ Média geral $\pm \mathrm{S}$} & $3966,0 \mathrm{a} \pm 603,3$ & $3961,0 \mathrm{a} \pm 606,6$ & $113,3 a+33,1$ & $110,0 b \pm 34,2$ & $0,438 \mathrm{a} \pm 0,05$ & $0,437 \mathrm{a} \pm 0,05$ & $2643,9 \mathrm{a} \pm 428,2$ & $2566,5 \mathrm{~b} \pm 421,8$ & $1208,9 a+258,3$ & $1178,8 b \pm 267,3$ \\
\hline
\end{tabular}

Médias seguidas por letras distintas na linha diferem entre si pelo teste $\mathrm{t}(\mathrm{P}>0,05)$.

CMO: conteúdo mineral ósseo; DMO: densidade mineral óssea: MM: massa magra, MG: massa gorda

$\mathrm{Na}$ Tab. 2, encontra-se expresso o CVt (\%) do pool de varreduras $(\mathrm{n}=35)$. Os valores de variâncias significativamente $(\mathrm{P}<0,05)$ maiores no grupo $\mathrm{CR}$ confirmam $\mathrm{O}$ efeito do reposicionamento e, portanto, a necessidade de incluí-lo na avaliação de precisão. Segundo Glüer et al. (1995), o erro de precisão pode ser subestimado em mais de $25 \%$ quando se utiliza apenas a média aritmética dos $\mathrm{CV}$, e pode explicar os valores de precisão mais elevados neste experimento em que se calculou o $\mathrm{CV}$ a partir da variância dos dados e erro quadrático médio. 
Tabela 2. Variância $\left(\mathrm{S}^{2}\right)$, coeficiente de variação da técnica $\left(\mathrm{CV}_{\mathrm{t}}\right)$ e intervalo de confiança (IC) do total de varreduras $(n=35)$ de corpo total de gatos adultos com (CR) e sem (SR) reposicionamento sobre a mesa de DEXA

\begin{tabular}{|c|c|c|c|c|c|}
\hline \multicolumn{6}{|c|}{ Variável } \\
\hline & Peso & $\mathrm{CMO}$ & DMO & MM & $\mathrm{MG}$ \\
\hline \multicolumn{6}{|c|}{$\mathrm{CR}$} \\
\hline $\mathrm{S}^{2}$ & $977,8 \mathrm{a}$ & $12,9 \mathrm{a}$ & $0,00006 \mathrm{a}$ & $12650,8 \mathrm{a}$ & $17435,2 \mathrm{a}$ \\
\hline $\mathrm{CV}_{\mathrm{t}}(\%)$ & 0,8 & 3,2 & 3,5 & 4,3 & 10,9 \\
\hline IC & $644,5-1871,8$ & $5,7-16,6$ & $0,0000-0,0001$ & $4193,4-12179,5$ & $6595,4-19155,7$ \\
\hline \multicolumn{6}{|c|}{ SR } \\
\hline $\mathrm{S}^{2}$ & $897,7 \mathrm{a}$ & $9,1 \mathrm{a}$ & $0,00024 b$ & $6601,7 b$ & $9877,8 b$ \\
\hline $\mathrm{CV}_{\mathrm{t}}(\%)$ & 0,7 & 2,8 & 1,8 & 3,2 & 7,7 \\
\hline $\mathrm{IC}$ & $634,3-1868,5$ & $9,4-27,2$ & $0,0001-0,0004$ & $6141,2-17836,6$ & $8116,4-23573,6$ \\
\hline
\end{tabular}

Valores seguidos por letras distintas na coluna diferem entre si pelo teste $\mathrm{F}(\mathrm{P}>0,05)$.

CMO: conteúdo mineral ósseo; DMO: densidade mineral óssea; MM: massa magra; MG: massa gorda.

Neste estudo, o GL obtido foi de 28GL, o que concorda com Glüer et al. (1995), que demonstraram ser adequado um número acima de 27GL. O uso apropriado do GL implicou erro de precisão ligeiramente mais elevado, em comparação a Munday et al. (1994) e Lauten et al. (2000), que não o estabeleceram. Segundo Glüer et al. (1995), a inclusão incorreta do GL pode subestimar em até $41 \%$ o erro de precisão da técnica.

O reposicionamento dos animais entre as varreduras gerou elevação dos valores médios de composição corporal, bem como dos CV, confirmando que o efeito dessa variável deve ser aliado ao do equipamento na determinação do erro de precisão em curto prazo. Para não subestimar esse erro, deve-se incluir o cálculo da variância dos dados, do erro quadrático médio e da estimação do IC e do GL. Nessas circunstâncias, a técnica DEXA demonstra ser precisa na estimativa da composição corporal em gatos adultos, e os resultados deste estudo asseguram a pesquisadores e clínicos a reprodutibilidade dos dados obtidos quando do emprego desta técnica.

\begin{abstract}
A short-term precision error of the individual subject and the DEXA technique, such as the effect of the repositioning of the cat on the examination table, were established. Four neutered adult cats $(B W=4342 \mathrm{~g})$ and three females $(B W=3459 \mathrm{~g})$ were submitted to five repeated scans with and without repositioning between them. Precision was estimated from the mean of the five measurements and expressed by the individual coefficient of variation $(\mathrm{CV})$. The precision error of the technique was estimated by the variance of scan pool $(n=35)$ and expressed in $C V$ for the technique $(C V t)$. The degrees of freedom and confidence intervals were determined to avoid underestimation of precision errors. Bone mineral content $(B M C)$, lean mass $(L M)$, and fat mass $(F M)$ averages were higher $(P<0.05)$ when animals were repositioned. The CVt was significantly higher $(P<0.05)$ for bone mineral density $(B M D)$, $L M$, and FM when the animals were repositioned. For short-term precision measurements, the repositioning of the animal was important to establish the precision of the technique. The dual energy $x$ ray absorptiometry method provided precision for body composition measurements in adult cats.
\end{abstract}

Keywords: cat, repeatability, DEXA

\section{AGRADECIMENTOS}

FAPESP, FUNDUNESP e Mogiana Alimentos S.A.

\section{REFERÊNCIAS BIBLIOGRÁFICAS}

BUSSAB, W.O.; MORETIN, P.A. Estatística básica. 5.ed. São Paulo: Saraiva, 2003. 520p. 
GLÜER, C.C.; BLAKE, G.; LU, Y. et al. Accurate assessment of precision errors: how to measure the reproducibility of bone densitometry techniques. Osteoporosis Int., v.5, p.262-270, 1995.

LAUTEN, S.D.; COX, N.R.; BAKER, G.H., et al. Body composition of growing and adult cats as measured by use of dual energy x-ray absoptiometry. Comp. Med., v.50, p.175-183, 2000.

MUNDAY, H.S.; BOOLES, D.; ANDERSON, $P$. et al. The repeatability of body composition measurements in dogs and cats using dualenergy x-ray absorptiometry. J. Nutr., v.124, p.2619S-2621S, 1994.

PIETROBELli, A.; FORMICA, C.; WANG, Z. et al. Dual-energy X-ray absorptiometry body composition model: review of physical concepts. J. Physiol., v.271, p.E941-E951, 1996.

SCHLOTZHAVER, S.D.; LITTLE, R.C. System for elementary statistical analysis. 2.ed. Cary, NC: SAS Institute, 1997. 441p. 